\title{
EMPIRISCHE ANALYSE EINER VERDOLMETSCHTEN GERICHTSVERHANDLUNG AUS DEM SLOWENISCHEN INS DEUTSCHE
}

\section{EINLEITUNG}

Das Recht, seine Muttersprache in den Gerichts- und anderen Verfahren vor den Staatsbehörden der Republik Slowenien benutzen zu dürfen, ist in der Verfassung verankert. Indem sie eine reibungslose und erfolgreiche Kommunikation zwischen dem Gericht und den Fremdsprechenden ermöglichen, tragen die Gerichtsdolmetscher effektiv zur Wahrung der Menschenrechte und des Grundsatzes des fairen Verfahrens bei und schaffen dadurch Gleichberechtigung zwischen allen Verfahrensbeteiligten (cf. Mikkelson 2000: 2). Um bei ihrer Arbeit erfolgreich zu sein, werden ihnen verschiedene Kompetenzen abverlangt - neben den allgemeinen Dolmetschfertigkeiten auch ein umfassendes juristisches Wissen sowie eingehende Kenntnisse der einschlägigen Rechtsordnungen. Während einer Verhandlung im Gerichtssaal werden aber auch andere Kompetenzen des Dolmetschers auf die Probe gestellt, indem nicht nur das Was, sondern auch das Wie sinngemäß in die Zielsprache transferiert werden soll. Diese Untersuchung hat das Ziel, den Diskurs einer verdolmetschten Zeugenvernehmung näher zu betrachten und herausfinden, ob und wie es dem Dolmetscher gelingt die im Ausgangstext geäußerten Aussagen in die Zielsprache zu übertragen. Die Ergebnisse der Untersuchung sollen eine Möglichkeit aufzeigen, wie die Kommunikationsteilnehmer im Gerichtssaal ihre Arbeits- und Kommunikationsweise verbessern, effizienter gestalten und so zu einem reibungsloseren Verfahrensablauf beitragen können. Außerdem soll der vorliegende Beitrag als Anregung für weitere Analysen zu diesem Thema dienen. Im ersten Teil wird zunächst der Rechtsdiskurs im Gerichtssaal theoretisch erörtert. Darauf folgend wird die konkrete Dolmetschtätigkeit im Gerichtssaal mit Bezug auf die Fachsprache und den Dolmetschmodus beschrieben. Anhand einer transkribierten gedolmetschten Zeugenvernehmung wird im vierten Teil eine empirische Analyse des Rechtsdiskurses im Gerichtssaal unternommen, die bei der Verdolmetschung festgestellten Abweichungen eingehender betrachtet und die Gründe dafür erörtert. Zuletzt werden noch künftige Perspektiven der Diskursanalyse im Gerichtssaal aufgezeigt.

\footnotetext{
*Adressen der Autoren: Lea Burjan, Cvetkova 33b Rakičan, 9000 Murska Sobota, Slowenien. Email: burjan.lea@gmail.com
} 


\section{2 ÜBER DEN RECHTSDISKURS IM GERICHTSSAL}

Im Gerichtssaal steht das gesprochene Wort im Vordergrund. Der Grundsatz der Mündlichkeit (Art. 4 slowenische ZPO) ${ }^{1}$ besagt, dass das Gericht sein Urteil lediglich darauf begründen darf, was bei der Hauptverhandlung entweder direkt, z. B. durch die mündliche Vernehmung von Zeugen oder Sachverständigen oder indirekt, z. B. durch das Vorlesen von Protokollen und Urkunden behandelt wird. Mit anderen Worten, man muss (persönlich) anwesend sein um „seine“ Wahrheit zu bekunden und sie gegenüber anderen Parteien zu verteidigen. Der Richter hat keinen Zugang zur fraglichen Wirklichkeit. Er muss aus dem, was erzählt oder berichtet wird, etwas machen, was rechtsförmig ist (Hoffmann 2007).

Urteile beruhen auf Gesetzen. Doch die Gesetze sprechen nicht, sie werden interpretiert. So liegt man mit der Annahme, es werde lediglich ein objektiv festgestelltes Geschehen dem Rechtssatz zugeordnet (,subsumiert“) und daraus ergebe sich logisch das Urteil, falsch. Die Wirklichkeit wird nicht so einfach zum Fall. Auch das Gesetz muss erst zum Sprechen gebracht werden (Hoffmann 2007). Die Realität im Gerichtssaal wird erst im Diskurs, im Medium der Sprache, (neu) aufgebaut und gegenseitig kommuniziert.

Bei einer Gerichtsverhandlung werden die Rechte der sprechenden Subjekte auf Teilnahme und Teilhabe am Rechtsdiskurs kanalisiert. Die Teilnahme am Verfahren gewährt die Möglichkeit an vorgesehenen Stellen sprechen und begründen zu dürfen. Dabei hat man bestimmte Äußerungsformen anzuwenden und sich an feste Kommunikationsregeln zu halten. Die Positionen der Sender und der Empfänger im Gerichtsraum sind räumlich bestimmt (Seibert 1994). Zeugen haben das Recht auf zusammenhängende Darstellung und Bekanntgabe des Themas. Da die Sprache im Recht zu einem Medium für Rechtsinstitutionen wird, treten die Vermittlungskompetenz der Diskursteilnehmer und die Sprache umso mehr ins Visier. Aus diesem Grund wird im Recht ständig an Sprache gearbeitet. Recht und Sprache stehen dabei in Wechselwirkung miteinander (Neumann-Duesberg 1949 zit. in Oksaar 1988: 56).

Der im Gerichtssaal stattfindende Rechtsdiskurs ist in mancher Hinsicht vorherbestimmt. Betrachtet man den gesamten Verfahrensablauf, so stellt man fest, dass der Diskurs im Gerichtssaal vom Aufruf der Sache, über die Eröffnung der Verhandlung und die Beweisaufnahme bis hin zur Urteilsverkündung, streng geregelt und seine effiziente Umsetzung dem personifizierten Vertreter des Gerichts, nämlich dem vorsitzführenden Richter, anvertraut ist. In einem Zivilverfahren leitet der Richter die Hauptverhandlung, führt Beweise aus, erteilt das Wort an die Senatsmitglieder, die Parteien, deren Rechtsbeistände und Bevollmächtigte und verkündet die Entscheidungen des Richtersenats (Art. 298 slowenische ZPO). ${ }^{2}$ Nicht viel anders übernimmt auch

\footnotetext{
14. člen Zakona o pravdnem postopku - Sodišče odloči o tožbenem zahtevku na podlagi ustnega, neposrednega in javnega obravnavanja.

2 298. člen Zakona o pravdnem postopku - Predsednik senata vodi glavno obravnavo, izprašuje stranke, izvaja dokaze, daje besedo članom senata, strankam ter njihovim zakonitim zastopnikom in pooblaščencem in razglaša odločbe senata.
} 
im Strafprozess der Richter die Rolle des moderierenden „Befehlshabers“, dessen Handlungen wohl im Gesetz und in der Verfassung begründet sein müssen. Er ist stets daraus ausgerichtet, die Wahrheit zu erkunden um darauf sein Urteil zu begründen.

Doch die Wahrheitssuche ist begrenzt, da der Prozess in vertretbarer Zeit und mit vertretbaren Kosten zu einem Ende kommen und die Streitfrage erledigt werden muss (etwa durch einen ausgehandelten Vergleich, eine Einstellung unter Auflagen oder durch ein Urteil) (Hoffmann 2007). Effizienz ist gefragt. Folglich wird dem Rechtsdiskurs im Gerichtssaal konsequente Formalität und begrenzte Möglichkeit der Diskursteilnahme abverlangt. Dadurch soll verhindert werden, dass Jedermann Zugang zum Rechtsdiskurs hat und (im Idealfall) auch, dass über Irrelevantes kommuniziert wird.

Viele Handlungsmuster werden im Gerichtssaal institutionell transformiert. In der Schule kennt der Fragende die Antwort, vor Gericht muss sich der Zeuge in der Antwort an die Wahrheit halten, während Angeklagte im Prinzip lügen dürfen oder auch zu den Vorwürfen schweigen können (Hoffmann 1992). Um die notwendigen Handlungen im Gerichtssaal effektiv umsetzen zu können, bedarf es einer gewissen Ordnung und Hierarchie. Die Dreier-Konstellation, „Richter-Kläger-Beklagte“, prädestiniert die Kommunikation, welche im Gerichtssaal strategisch bestimmt sein muss, um lediglich das Zielführende zum Ausdruck bringen zu lassen. Dem Richter kommt eine übergeordnete Rolle zu, andere Gesprächsteilnehmer sind seinen Weisungen unterstellt und haben diese zu akzeptieren um an der Kommunikation weiter teilnehmen zu dürfen.

\section{3 ÜBER DIE DOLMETSCHTÄTIGKEIT IM GERICHTSSAAL}

Die bisher geschilderte Interaktion im Gerichtssaal wird durch die Hinzuziehung eines Gerichtsdolmetschers neu definiert. Die Präsenz des Dolmetschers im Gerichtsverfahren muss nämlich stets als ein eindringliches Element angesehen werden. Das manifestiert sich in vielerlei Hinsicht - von der bloßen Präsenz des Dolmetschers im Gerichtssaal, der entsprechenden Platzzuweisung und Vorstellung seiner Person gegenüber den Geschworenen, Anwälten uä., über die allgemein verbreitete Praxis mancher Richter und Anwälte, statt den Zeugen den Dolmetscher zu adressieren, bis hin zu der Notwendigkeit, dass vom Dolmetscher bestimmte Fragestellungen der Anwälte oder Aussagen des Zeugen zusätzlich erläutert werden müssen. (Berk-Seligson 2002: 96). Nicht selten löst der Dolmetscher durch seinen Auftritt im Gerichtssaal zusätzliche Besorgnis hinsichtlich des Kommunikationsverlaufs aus. Außerdem spielt er in diesem Sprechakt oft eine entscheidende Rolle, indem er die Rede des Zeugen oder des Beklagten, dessen Aussage er dolmetscht, kontrolliert (Berk-Seligson 2002: 86) Diese Kontrolle wird durch die Anwendung verschiedener Mechanismen, mittels derer der Befragte entweder zum Reden aufgefordert oder zum Schweigen gezwungen werden kann, deutlich.

Obwohl der Dolmetscher, wie bereits erwähnt, den Weisungen des Richters unterworfen, also kommunikativ untergeordnet ist und seine Aufgabe in erster Linie in der wortwörtlichen Wiedergabe der im Gerichtssaal geäußerten Aussagen besteht, so wird an ihn gleichzeitig auch die Forderung gestellt, dass er auch die Gesprächsstrategie, den 
tatsächlichen Sinn und die wahre Intention der Aussagen vor Gericht möglichst originaltreu in die Zielsprache transferiert, also (zumal nur in der Fremdsprache) kommunikativ gleichwertig zu fungieren versucht. Ein Widerspruch in sich selbst, der bereits von mehreren Forschern aufgegriffen wurde. So behauptet Mikkelson, dass der Dolmetscher nichts hinzufügen, ergänzen, erläutern oder erklären darf (Mikkelson 1996: 2), noch mehr, dass es nicht seine Aufgabe sei, zu gewährleisten, dass der Beklagte das Verfahren versteht (Mikkelson 1996: 2). Auch Morris (Morris 1995) bezweifelt, ob diese beiden Kriterien überhaupt gleichzeitig erfüllt werden können. Tatsache ist, dass sich der Dolmetscher während seiner Arbeit immer gezwungen sieht, in der Spannweite zwischen diesen beiden Extremen das richtige Gleichgewicht herzustellen.

\subsection{Fachsprache und Sprachregister}

Die Juristen benutzen in ihren Ausführungen großenteils die Alltagssprache; dies ist erforderlich, weil die Rechtstexte oft nicht nur an Experte gerichtet sind und, weil sie auch für rechtsunkundige Adressaten, also ein weiteres Publikum, verständlich sein müssen. Andererseits ist die juristische Sprache aber auch eine Fachsprache. Die wesentlichen Charakteristika der Fachsprache sind eine charakteristische Auswahl, Verwendung und Frequenz der Sprachmittel, vor allem auf der morphologischen, lexikologischen, syntaktischen und textuellen Ebene, (Möhn/Pelka 1984: 26), ein minderer Grad der Abhängigkeit von der Individualität des Kommunikators (Von Hahn 1983: 68) und ein spezifischer, den Bedürfnissen des jeweiligen Fachbereiches angepasster Wortschatz, der fließend in die Allgemeinsprache übergeht und darüber hinaus allgemein verständliche Wörter aus der Alltagssprache enthält (Hoffmann 1985: 53).

Die wichtigsten Merkmale der juristischen Fachsprache sind Abstraktheit, Schlichtheit, Nüchternheit, Knappheit, Ausführlichkeit, Technizität und Mehrdeutigkeit von Rechtsbegriffen in verschiedenen Rechtsgebieten. Des Weiteren werden in der Gesetzessprache juristische Fachausdrücke verwendet, deren Bedeutung gelegentlich nicht oder nur teilweise mit dem alltäglichen Sprachgebrauch übereinstimmt.

Auf jeden Fall ergibt sich aus dem spezifischen Gebrauch der Sprache im Gerichtssaal eine Variation der Sprache. Halliday (Halliday 1978) bezeichnet diese als Register und ist davon überzeugt, dass, die Relevanz des Registerkonzeptes, vor allem für die Übersetzung in der Tatsache besteht, dass es als eine Art „prediction“ verstanden wird, so dass aus der Situation, die Auswahl der sprachlichen Formen, die darin funktionieren, vorhergesagt werden kann (Halliday 1978: 32). Diese Vermutung oder Antizipation muss auch der Dolmetscher bei seiner Tätigkeit entsprechend einfließen lassen.

\subsection{Dolmetschmodus}

Es gibt mehrere Dolmetschtechniken, jedoch sind im Gerichtssaal bei der Zeugenvernehmung die nachstehenden drei Dolmetschmodi praxisüblich. 


\section{Konsekutivdolmetschen}

Bei diesem Modus überträgt der Dolmetscher das Gesagte in eine andere Sprache, nachdem der Redner seine Ausführungen beendet hat. Diese Verdolmetschung geschieht ohne den Einsatz von technischen Hilfsmittelt (die beim Simultandolmetschen zur Anwendung kommen) und fordert den größten Zeitaufwand. Das Konsekutivdolmetschen wird am häufigsten bei den Gerichtsverhandlungen und Zeugenvernehmungen verwendet. Dieser Modus ist nicht ohne Grund der am meisten verbreitete, er erlaubt nämlich als einziger die kontinuierliche Prüfung, ob der Fremdsprechende, das gesagte auch (richtig) verstanden hat und gewährt die Möglichkeit, im entgegengesetzten Fall sofort in die Kommunikation einzugreifen um etwaige Korrekturen vorzunehmen.

\section{Flüsterdolmetschen}

Das Flüsterdolmetschen, im Vergleich zum Konsekutivdolmetschen, passiert ohne den Kommunikationsablauf zwischen den Hauptakteuren im Gerichtssaal zu unterbrechen. Laut Kalina (Kalina 1998) wird Flüsterdolmetschen oft als erschwertes Simultandolmetschen bezeichnet, weil die zeitliche Überlappung die gleiche ist, die Hör- und Sprechbedingungen aber mangels geeigneter technischer Einrichtungen schlechter sind. Die Rezeption des Dolmetschers wird (vorwiegend negativ) durch zusätzliche Faktoren, wie Klangfarbe, Stimmstärke, Akzentuierung uä. mitbeeinflusst. An dieser Stelle sei auch erwähnt, dass Flüsterdolmetschen und das Vom-Blatt-Dolmetschen (siehe weiter unten), die in der Praxis des Dolmetschens vor Gericht eine bedeutende Rolle spielen, weitgehend unerforscht sind. Ein gewichtiger Grund hierfür ist ganz bestimmt die Tatsache, dass sich die Aufnahme des zu untersuchten geflüsterten Zieletextes als zu aufwendig versteht.

Man unterscheidet zwei Modi des Flüsterdolmetschens. Beim ersten befindet sich der Dolmetscher hinter maximal zwei fremdsprechenden Personen und spricht ihnen simultan die Übersetzung des Gesagten leise ins Ohr. Diese Dolmetschtechnik kommt im Gerichtssaal immer dann zum Einsatz, wenn der Kommunikationsfluss durch die Verdolmetschung nicht unterbrochen, wohl aber den Fremdsprechenden der Kommunikationsablauf und somit der Verhandlungsablauf deutlich und verständlich gemacht werden soll.

Beim zweiten Modus benutzt der Dolmetscher eine sogenannte Infoport- oder Flüsteranlage, in welche er hinein flüstert und seine Verdolmetschung an mehr als zwei Zuhörer gleichzeitig übertragen wird. Diese Dolmetschtechnik wird vor allem bei gröBeren Prozessen mit mehreren Angeklagten angewandt.

\section{Vom-Blatt-Übersetzen}

In bestimmten Situationen ist es nicht das gesprochene Wort, sondern ein schriftlicher Text, der von dem Dolmetscher in die jeweilige Zielsprache (mündlich) übertragen wird. Diese Technik, genannt Vom-Blatt-Übersetzen, wird eingesetzt, wenn im Gerichtssaal fremdsprachige Urkunden und Gutachten oder Protokolle (z. B. Proto- 
kolle der Polizei) verlesen werden. Dieser Art von Dolmetschen wird in der vorliegenden Untersuchung kein besonderes Augenmerk geschenkt.

Welche Dolmetschtechnik bei der Gerichtsverhandlung eingesetzt werden soll, wird grundsätzlich vom Gericht beschlossen, dem Dolmetscher im Gerichtssaal jedoch nur indirekt vorgegeben. Aufgrund mangelnder technischer Ausstattung wird in der Regel konsekutiv gedolmetscht. Simultandolmetschen wird nur in Form von Flüsterdolmetschen durchgeführt. Manchmal muss der Dolmetscher während der Verhandlung selbstständig den Modus wechseln. Dies ist vorwiegend der Fall bei Einsprüchen, wenn Teile der Aussagen im Gerichtssaal sich zeitlich überlappen. In solchen Situationen greift der Dolmetscher auf sein verbales Werkzeug und Kontrollmechanismen zurück. So deutet er zum Beispiel mit bestimmten Äußerungen darauf hin, dass Informationen wiederholt werden sollen, oder wechselt eben, wenn der Redefluss zu schnell geworden ist, in den Simultanmodus, um Zeit zu gewinnen und weiterhin eine entsprechende Verdolmetschung gewährleisten zu können. Inwiefern die Anwendung dieser Mechanismen die Perzeption des Richters gegenüber dem Zeugen oder Angeklagte beeinflusst, bleibt offen, allerdings verursachen Einsprüche, die im Rahmen einer gedolmetschten Zeugenvernehmung erhoben werden - zumindest für den Dolmetscher und den auf ihn angewiesenen Fremdsprechenden - eine sehr konfuse Situation. Sobald der erste Anwalt eine Frage stellt, erhebt der andere Einspruch und bis der Dolmetscher die erste Frage an den Zeugen verdolmetscht hat, hat der Richter bereits dem Einspruch stattgegeben oder ihn zurückgelehnt (nach Berk-Seligson 2002: 91).

\section{EMPIRISCHE ANALYSE DER VERDOLMETSCHUNG DES RECHTSDISKURSES IM GERICHTSSAAL}

\subsection{Zur Transkription und der Ausgangssituation}

Der empirische Teil der wissenschaftlichen Auseinandersetzung mit diesem Thema wurde in Februar 2012 am Bezirksgericht in Maribor durchgeführt. Da slowenische Gerichte in letzten Jahren vermehrt Audioaufnahmen von mündlichen Verhandlungen veranlassen, konnte auf diese zurückgegriffen werden um entsprechende Textkorpora für die Untersuchung zu gewinnen. Dies erleichterte die Arbeit sehr, da aufwendiges und technisch kompliziertes Aufnehmen im Gerichtssaal nicht notwendig gewesen war. Bei der Aufnahme handelt es sich um eine offene Aufnahme, die angekündigt und im Anschluss daran durchgeführt wurde. Die Verdolmetschung wird von einem gerichtlich beeidigten Dolmetscher angefertigt. Die Gesamtlänge des Gesprächs beträgt 14:45 Minuten.

Es wurde orthographische Transkription verwendet wie sie in Brinker und Sager beschrieben wird. „Der Vorteil dieses Verfahrens besteht darin, dass es leicht lesbar und leicht anwendbar ist, gleichzeitig aber die kommunikativ relevanten Besonderheiten der gesprochenen Sprache erfasst werden“" (Brinker/Sager 2010). Alle personen- und firmenbezogenen Daten wurden aufgrund datenschutzrechtlicher Bestimmungen verändert.

Die bei der Transkription verwendeten Zeichen haben folgende Bedeutungen: 


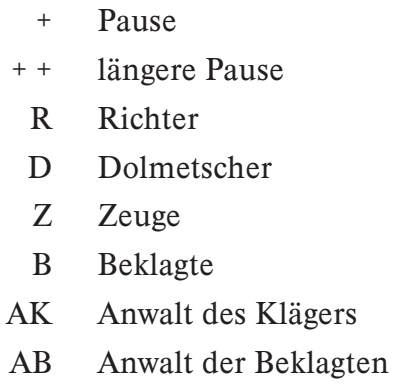

Wortlaut (grau unterlegt) gleichzeitiges Sprechen mehrerer Gesprächsteilnehmer \& simultanes Flüsterdolmetschen

Wortlaut (grau unterlegt \& simultanes Flüsterdolmetschen

Kursivschrift)

Wortlaut (Kursivschrift) schlechter hörbare Wörter oder Passagen

Die untersuchte Konversation wird als Gesprächstyp Vernehmung klassifiziert. Das Thema und gleichzeitig das Ziel der vorliegenden Besprechung ist die Befragung des Zeugen zu einem vorher bekannten Sachverhalt. Demzufolge widmet sich das vorliegende Gespräch vor allem dem Zweck des Informationsaustausches und der Entscheidungsvorbereitung. Es bildet die Grundlage für den weiteren Verlauf und mögliche Handlungen im Gerichtsverfahren.

Der gewählte Dolmetschmodus ist konsekutiv. In der letzten Minute des Gespräches, wenn vom Anwalt der klagenden Partei Einsprüche erhoben werden, wechselt der Dolmetscher ohne Aufforderung in den Simultanmodus und dolmetscht flüsternd. Ausgelöst durch neue Kommunikationsbedingungen (Überlappung der Aussagen, schneller Wechsel der Gesprächsteilnehmer), wählt der Dolmetscher den rationellen Entschluss, die Verdolmetschung, sobald Einspruch erhoben wird, vorerst zu unterbrechen. Um dem Zeugen weiterhin die Teilhabe am Geschehen zu ermöglichen, entschließt er sich kurz darauf flüsternd dolmetschend fortzufahren. Weil situative Faktoren (schlechter akustischer Empfang, erhöhter Stressfaktor, intensive Belastung der Stimmlippen, das physische und psychische Wohlbefinden) bei diesem Modus eine noch größere (negative) Auswirkung auf den Dolmetscher entfalten, ist die Verdolmetschung in diesem Teil der Konversation deutlich unvollständiger.

\subsection{Analyse}

Bei der Analyse wird nach Henne und Rehbock (Henne/Rehbock 2001: 14) vorgegangen und dabei die folgenden Kategorien am konkreten Beispiel näher beleuchtet:

\subsubsection{Kategorien der Makroebene: Gesprächsphasen}

\section{a. Gesprächseröffnung}

Die äußere Struktur eines Gespräches gliedert sich in drei Hauptteile: die Einleitung, den Kern und den Schluss (Levinson 1983, übernommen nach Verdonik 2007). 
In unserem Fall stellt die Aufnahme lediglich den Kern des Gesprächs dar. Eingeführt wird das Gespräch durch eine entsprechende Begrüßungsformel. Da aus der Aufnahme darüber nichts Konkretes vorliegt, könnte man als den ersten Sprechakt bereits den Aufruf der Sache verstehen, auf den der Gerufene/der Geladene so reagiert, dass er den Gerichtssaal betritt. Im ersten thematischen Abschnitt wird jedenfalls der Kontakt zwischen den Gesprächspartnern aufgenommen, indem zuerst die Personalien der geladenen Person nach einem vorherbestimmten Fragenkatalog abgefragt werden und der zu Befragende eine Rechtsbelehrung erteilt bekommt. Das sind feste Bestandteile der Zeugenvernehmung, die auch gesetzlich geregelt sind. Den Beginn des Kernteiles signalisiert der Beginn der Audioaufnahme, der vom Richter angeordnet wird. Der Dolmetscher erhält zu Beginn seiner Tätigkeit im Gerichtssaal keinerlei Weisung bezüglich des Dolmetschmodus, dieser ergibt sich automatisch aus der vorgefundenen Situation (es gibt keine technische Ausstattung im Saal, der Dolmetscher wird mit dem Zeugen zum Rednerpult aufgefordert). Weil die Vernehmung aufgenommen wird, wird er, zusammen mit dem Vernommenen, aufgefordert in das Mikrofon zu sprechen und unnötige Geräusche zu vermeiden.

\section{b. Gesprächsmitte (Entfaltung des Themas und der Subthemen)}

Das Hauptthema ist die Befragung über einen bestimmten Sachverhalt, nämlich die Existenz von verschiedenen Verträgen und sich daraus ergebenden Rechtsverhältnissen. In der zentralen Sektion des Gespräches werden verschiedene Subthemen in der Regel nach dem Frage-Antwort Prinzip besprochen, und zwar so lange, bis, nach dem Ermessen des Gerichtes, das erwartete Informationsniveau erreicht und die Fragen ausgeschöpft sind.

\section{c. Gesprächsbeendigung (Nebenthemen, Episoden)}

Nebenthemen treten lediglich in den Fragen auf, die im letzten Teil der Konversation seitens der Verteidigung bzw. der Beklagten selbst gestellt werden. Der Abschluss der Konversation wird sehr abrupt durch den Richter herbeigeführt und signalisiert gelichzeitig das Ende der Audioaufnahme. Danach wird nur noch die Erstattung von Kosten besprochen und der Zeuge und der Dolmetscher mit einer Abschiedsformel entlassen.

\subsubsection{Kategorien der mittleren Ebene}

\section{a. Sprecher-wechsel (turn taking)}

Schon vor Gesprächsbeginn ist wegen der institutionellen Rollenverteilung und Hierarchie im Gerichtssaal klar, dass es sich um ein Gespräch mit einer Rollentrennung in „bevorrechtigte“ und „nicht-bevorrechtigte" Partner handelt. Die Gesprächseröffnung erfolgt durch den Richter. Er ist es auch, der die Kernphase thematisch einleitet und bestimmt. Bereits mit der Eröffnungsfrage gibt er zu verstehen, dass man ihm zuhören und seiner Gesprächsstrategie folgen solle. So beginnt er seine Befragung mit dem einleitenden Fragesatz, 
RICHTER:

DOLMETSCHER:
Najprej bi gospoda vprašal, eh, ... kaj če in kaj mu je znano glede Zuerst möchte ich Sie, Herr Müller, fragen, ob Ihnen was bekannt ist, beziehungsweise, was ist Ihnen bekannt, bezüglich

welches als initiierendes Startsignal betrachtet werden kann und auf das ein Hörersignal auf verbaler oder nonverbaler Art erwartet wird. Es scheint ein grundsätzlicher Respekt vor dem Wort des Ranghöchsten zu existieren. Andere Teilnehmer warten, bis die Frage zu Ende formuliert ist, bevor das Wort von dem Befragten ergriffen wird. Um zu Wort kommen zu dürfen, muss zuerst das Rederecht erteilt werden. Dies erfolgt, mit einer Ausnahme, in allen Fällen durch Fremdwahl, die dem bevorrechtigten Gesprächspartnern, in einer Gerichtsverhandlung also dem Richter, obliegt. Weil das Rederecht bewusst erteilt wird, vollzieht sich ein glatter Sprachwechsel. Nur einmal bittet der Zeuge etwas ergänzen zu dürfen und ergreift so die Initiative im Gespräch. Der Dolmetscher folgt in seinen Ausführungen konsequent dem Fragestellungs-Modus des Gesprächsmoderators und schlüpft nur einmal spontan in seiner Rolle, indem es den Zeugen nach einer bestimmten Information hinterfragt.

$\begin{array}{ll}\text { RiCHTER: } & \text { Mhm. Lahko pove mogoče ime gospoda Žigarta? } \\ \text { DolmetSCHER: } & \text { Könnten Sie den Namen von Hern Žigart nennen? Den } \\ \text { Namen... } & \text { Žigart. } \\ \text { ZeUGE: } & \text { Žigart ist der Nachname, den Vornamen. } \\ \text { DolmETSCHER: } & \text { Nein. } \\ \text { ZeUGE: } & \text { Ne, ne vem. }\end{array}$

Es gibt im ganzen Gespräch lediglich eine Unterbrechung durch den Richter. Diese erweist sich als notwendig, wenn er ein Lapsus in der Frage der Beklagten entdeckt sich entschließt unmittelbar in die Kommunikation einzugreifen, um zu korrigieren. Die Unterbrechung findet unmittelbar nach der Fragestellung und vor der Verdolmetschung statt und hat eine ausschließlich aufklärende und korrigierende Intention. Den Dolmetscher unterbricht er zu keinem Zeitpunkt. Insofern gibt es auch keine Anzeichen dafür, dass die Verdolmetschung, nämlich deren Qualität oder Vollständigkeit in irgendwelcher Weise unter Frage gestellt würde. Vielmehr bejaht der Richter in seinen Erwiderungen die verdolmetschten Aussagen und baut auf der bereits vorhandenen Information seine nächste Frage auf. Die Bejahung wird durch verschiedene Wörter ausgedrückt:

Richter: $\quad \underline{Z}$ e, ampak zanima me, kdo se je pred gospodom ukvarjal s temi pogodbami?

Richter: $\quad$ Aha, zdaj, glede na to, da je gospod prevzel ta spis šele v mesecu marcu ...

RICHTER: $\quad$ Mhm. Lahko pove mogoče ime gospoda?

RiCHTER: $\quad \underline{\mathrm{Mhm}}$. Eh, nam lahko poveste mogoče vsebino razgovora.

RichTER: $\quad$ Mhm. Prav. Gospod odvetnik, izvolite. 
Auch hierdurch wird die höhere Stellung des Richters in dem Gespräch deutlich. Mit dem bejahenden Partikel schließt er das Teilthema ab und eröffnet ein neues oder bewegt sich mehr in die Tiefe der Aussage. Das Recht eröffnende und beendende Gesprächsschritte durchzuführen sind eindeutig auf die Rolle des Richters geknüpft. Die ranghöchste Person ist die gesprächsleitende Person. Sie hat das Recht Gesprächssequenzen sowohl zu eröffnen, als auch zu beenden. Nur wenn diese Rollenverteilung von allen akzeptiert wird, kann eine Besprechung erfolgreich verlaufen. Allerdings ist die Kohäsion im thematischen Aufbau der Fragestellungen in der Verdolmetschung nicht so deutlich ausgeprägt,

DOLMETSCHER: Wer hat sich also konkret vor Ihnen mit dieser Akte und mit diesen Mietverträgen beschäftigt?

DolmetscheR: Hinsichtlich der Tatsache, dass Sie in März dieses Jahres die Akte beziehungsweise diesen Fall übernommen haben,

DOLMETSCHER: Könnten Sie den Namen von Herrn Žigart nennen?

Dolmetscher: Könnten Sie uns den Inhalt dieses Gespräches darlegen?

trotzdem kann man behaupten, dass aufgrund der äquivalenten Übersetzung des Frageninhalts die Konversation dadurch weder gestört noch inhaltlich beeinflusst wird.

Während der Zeuge bis zu einem gewissen Grad seine Aussage unter Kontrolle behält, liegt die linguistische Macht beim Richter und dem Anwalt. Im gegebenen Kontext werden Fragen mehr als das: sie werden zu Befehlen, Aufforderungen, dass das Wissen des Befragten auf eine entsprechende Weise dargelegt werden soll (nach Berk-Seligson 2002: 23). Die Fragen sind ausführlich, relativ lang und veranlassen den Zeugen dazu im narrativen Stil zu antworten. Auf die initiierenden Gesprächsakten des Richters folgen konsequent responsive Reaktionen des Zeugen. Der Zeuge tendiert dazu relativ lange Aussagen als Antworten anzuführen. Die Aussagen werden durchgehend, ohne Unterbrechung des Dolmetschers, in die Zielsprache transferiert. Dies trägt zu einer kompakten Informationsübermittlung bei, es gehen keine Informationen verloren, da die gedolmetschten Aussagen in der Regel (wie später noch dargestellt wird) mehr erläuternde Elemente enthalten, als deren Originale.

Das Frage-Antwort-Prinzip, das dem gesamten Gespräche zu Grunde liegt, zeigt eine klare Gesprächshierarchie. Die vom Richter gestellten Fragen sind stets als initiierende Schritte zu betrachten, die einen respondierenden Schritt seitens des Hörers einfordern. Der Richter wird dadurch zum Gesprächsleiter, der das Gespräch lenkt und kontrolliert. Er stellt die Fragen, erwartet Antworten und determiniert so den Gesprächsverlauf.

\section{b. Gesprächssequenz}

Schon bei der ersten Betrachtung stellt man fest, dass die Mehrheit der gedolmetschten Aussagen in der Regel länger ist, als die ausgangssprachlichen Aussagen. Das gilt für die Sprachpaare Richter-Dolmetscher und Zeuge-Dolmetscher. Die allgemeine Annahme, dass eine slowenische Übersetzung in der Regel, wegen dem syntaktischen Aufbau, länger ist als das deutsche Original, wird hier bestätigt, weil im 
Sprachpaar Zeuge-Dolmetscher, wo ins Slowenische gedolmetscht wird, der Grad der Aussagenverlängerung um 7\% höher ist als bei der Verdolmetschung ins Deutsche. Andererseits sind die Aussagen des Anwalts und der Beklagten länger als deren Verdolmetschung. Dies ist zurückzuführen, im Fall des Anwalts, auf seinen ausgesprochen ruhigen und langsamen Redestil und im Fall der Beklagten, auf deren unsichere und unverständliche Fragestellung, ausgelöst dadurch, dass sie von ihrem Rechtsbeistand allein im Gerichtssaal zurückgelassen wurde und plötzlich gezwungen war, dessen Rolle zu übernehmen und Fragen an den Zeugen zu stellen. Eine Übersicht der Länge der einzelnen Aussagen bietet die Tabelle 1.

\begin{tabular}{|c|c|c|c|c|c|c|c|c|c|c|}
\hline $\begin{array}{c}\text { SPRE- } \\
\text { CHER }\end{array}$ & \multicolumn{7}{|c|}{} & $\begin{array}{c}\text { GE- } \\
\text { SAMT }\end{array}$ & $\begin{array}{c}\text { LANGE } \\
\text { IN } \%\end{array}$ \\
\hline R & $00: 25: 10$ & $00: 06: 40$ & $00: 06: 50$ & $00: 15: 00$ & $00: 04: 20$ & $00: 08: 70$ & $00: 06: 00$ & $00: 07: 80$ & $01: 19: 70$ & \\
\hline D & $00: 26: 00$ & $00: 03: 50$ & $00: 04: 50$ & $00: 12: 00$ & $00: 03: 10$ & $00: 08: 50$ & $00: 04: 00$ & $00: 02: 90$ & $01: 04: 50$ & $+14 \%$ \\
\hline Z & $00: 51: 00$ & $00: 13: 10$ & $00: 05: 70$ & $00: 07: 60$ & $00: 02: 20$ & $00: 01: 20$ & $00: 37: 30$ & $00: 14: 00$ & $03: 47: 70$ & \\
\hline Z & $00: 31: 10$ & $00: 01: 60$ & $00: 25: 90$ & $00: 02: 30$ & $00: 15: 50$ & $00: 19: 20$ & & & & \\
\hline D & $00: 54: 40$ & $00: 19: 70$ & $00: 05: 40$ & $00: 04: 80$ & $00: 02: 50$ & $00: 01: 60$ & $00: 44: 90$ & $00: 14: 40$ & $04: 20: 70$ & $+21 \%$ \\
\hline D & $00: 40: 30$ & $00: 01: 20$ & $00: 25: 80$ & $00: 02: 70$ & $00: 18: 80$ & $00: 24: 20$ & & & & \\
\hline AB & $00: 26: 30$ & $00: 21: 30$ & $00: 07: 80$ & $00: 01: 60$ & & & & & $00: 57: 00$ & $+6 \%$ \\
\hline D & $00: 26: 60$ & $00: 17: 10$ & $00: 08: 30$ & $00: 01: 40$ & & & & & $00: 53: 40$ & +60 \\
\hline B & $00: 06: 20$ & & & & & & & & $00: 06: 20$ & $+24 \%$ \\
\hline D & $00: 05: 00$ & & & & & & & & $00: 05: 00$ & \\
\hline
\end{tabular}

Tabelle 1: Länge der Aussagen

Es wird nachstehend versucht herauszufinden worauf die Aussagenverlängerungen in den einzelnen Gesprächssegmenten zurückzuführen sind. Stanek gibt al ein Hauptgrund der Aussagenverlängerung bei der Verdolmetschung an, dass praktizierende Dolmetscher Mühe mit der (Rechts-)Terminologie haben, was auf die fehlende fachspezifische Ausbildung zurückzuführen ist und wurde bereits wissenschaftliche erkannt wurde (Stanek 2011). Allein unter diesen Umständen ist deshalb nachvollziehbar, dass eine Verdolmetschung länger ausfällt als das Original. Wiederholungen oder Umschreibungen können zusätzlich verstärkt auftreten, wenn der Dolmetscher aufgrund mangelnder Vorbereitung selbst unsicher ist oder der Beschuldigte den Eindruck macht, das Gesagte nicht zu verstehen. (Stanek 2011)

In unserem Fall kann festgehalten werden, dass einzelne Fachtermini und Syntagmen (wie z. B. zavrniti - zurückweisen, fizična oseba - natürliche Person, trditvena podlaga - Behauptungsgrund) bei der Verdolmetschung problemlos und ordnungsgemäß in die Zielsprache übertragen werden. Offensichtlich verfügt der Dolmetscher über das notwendige Fachwissen. Es zeigt sich einmal mehr, dass die Sprache dem Juristen nicht als Fachsprache zum Problem wird, sondern dann, wenn sie den Fachkreis ver- 
lässt. Im Rechtsverfahren muss nämlich die Fachsprache den Bereich der Primärsprache verlassen um in der Praxis umgesetzt werden zu können (Oksaar 1988: 87). Andererseits zeigt sich in den vorliegenden verdolmetschten Aussagen eine starke Tendenz zu Ergänzungen bzw. Veränderungen, die im Wesentlichen eine Verlängerung der Verdolmetschung bewirken. Die nähere Betrachtung weist folgende Ergänzungen in der Verdolmetschung gegenüber dem Ausgangstext auf.

Der Dolmetscher fügt Höflichkeitsformeln, in Form von direkter Anrede, hinzu,

RICHTER: $\quad$ Najprej bi gospoda vprašal ...

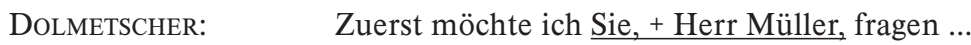

tendiert zu unnötigen Wiederholungen,

RichteR: $\quad$... če in kaj vam je znano glede pogodbe ...

DOLMETSCHER: $\quad$... ob Ihnen was bekannt ist, beziehungsweise, was ist Ihnen bekannt bezüglich der Verträge ...

\section{zusätzlichen Erklärungen und Umschreibungen}

RichteR: Und selbst für den Fall, den ich hier dezidiert ausschließen möchte, dass sie eine Zusage gemacht hätte, ...

DOLMETSCHER: In tudi v primeru, ki pa ga želim na tem mestu še enkrat izrecno zanikati, ampak tudi v primeru če bi to bilo narejeno, če bi to soglasje z njene strani bilo dano, ...

oder

Zeuge: $\quad$ Es ist aber so innerhalb des Konzernes, dass wir immer die Zustimmung der Geschäftsführung brauchen.

Dolmetscher: Vendar je pri nas znotraj koncerna tako, da velja pravilo, da za vse postopke, $\underline{\text { ki jih vodimo, }}$ potrebujemo potrdilo predstojnika, torej oddelka, ali poslovodje našega, nadrejenega.

oder sogar zur Hinzufügung neuer Informationen

RICHTER:

... in pa, eh, Topič Tanjo in pa Nevenko Žigart.

DOLMETSCHER:

... und dann noch die natürlichen Personen, Tanja Topič und Nevenka Žigart.

RICHTER: Isto vprašanje velja za gospo Paulo Ekarim.

DOLMETSCHER. Es gilt jetzt nochmal die gleiche Frage, bezogen nicht auf Ihren Vorgesetzten, sondern auf Paula Ekarim.

höchstwahrscheinlich mit der Absicht, dem Befragten die Frage verständlicher zu machen oder die Aussage ausführlicher zu interpretieren. Ob dadurch die Aussage für 
den Rezipienten wirklich verständlicher ist, bleibt offen, auf jeden Fall wäre eine derartige Ausdehnung der Mehrheit der Aussagen unberechtigt, einerseits gegenüber dem Gesprächsmoderator, weil es seine Ausdrucksart verzerrt und andererseits gegenüber dem Befragten, weil es ihn in eine unterworfene, offenbar stets erklärungsbedürftige Rolle zwingt. Interessant ist, dass diese Ergänzungen nur in längeren Passagen vorkommen, wogegen bei kürzeren Aussagen einerseits die Höflichkeitsformeln oder sogar Teile der Information durch die Verdolmetschung komplett wegfallen können.

RICHTER: $\quad$ Če nam lahko pove prosim, eh, kdo je bil njegov predhodnik?

DOLMETSCHER: Wer war Ihr Vorgänger?

RICHTER: Zanima me, ali, oziroma, kako je gospod Žigart na to reagiral?

DOLMETSCHER: Wie hat Herr Žigart darauf reagiert?

Hier sieht der Dolmetscher offenbar keinen Bedarf Aussagen zusätzlich erläutern zu müssen.

\subsubsection{Kategorien der Mikroebene}

Sprechaktinterne Elemente: syntaktische, lexikalische, phonologische und prosodische Struktur

Die am meisten ausgeprägte Abweichung auf der syntaktischen Ebene zeigt sich in den Anredesequenzen. Konsequent adressiert der Richter in seinen Fragestellungen den Dolmetscher, anstatt den Zeugen, wobei der Dolmetscher dies in seiner Verdolmetschung korrigiert und die direkte Anrede wählt.

RichteR: $\quad$... Če nam lahko (on) pove prosim, eh, kdo je bil njegov predhodnik?

Če ga lahko (on) imenuje?

DOLMETSCHER: ... Wer war Ihr Vorgänger?

Könnten Sie den Namen nennen?

In diesem Fall kann man sogar behaupten, dass sich der Dolmetscher in seiner Rolle des Intervenierenden (ohne Erlaubnis und Zustimmung des Gesprächsleiters) über die Rolle des Gesprächsleiters erhebt um diesen zu korrigieren. Das Ziel dieser Intervention liegt allerdings in der klaren Hervorhebung der Rollenverteilung, in welcher der Gesprächsleiter auf andere Gesprächsteilnehmer initiierend einzuwirken versucht.

\section{FAZIT UND PERSPEKTIVEN DER DISKURSANALYSE IM GERICHTSSAAL}

Die situative Analyse einer gedolmetschten Zeugenvernehmung hatte sich zum Ziel gesetzt, anhand einer empirischen Analyse einer gedolmetschten Zeugenvernehmung herauszufinden, inwiefern es dem Gerichtsdolmetscher gelingt, den ausgangssprachlichen Diskurs originaltreu in die Zielsprache zu übertragen und auf welche Probleme er bei diesem Übertragungsprozess stößt. Anhand der Ergebnisse dieser Arbeit können folgende Schlussfolgerungen festgehalten werden. 
- Der Dolmetscher gestaltet durch seine Präsenz und Handlungen den stark strukturierten und institutionalisierten Diskurs im Gerichtssaal aktiv mit.

- Da er nicht nur die/den Befragten sondern auch den Fragenden dolmetschen muss, ist er der Gesprächsteilnehmer mit dem größten Gesprächsanteil im gedolmetschten Rechtsdiskurs im Gerichtssaal, allerdings nicht derjenige mit den meisten Gesprächsrechten.

- Der Dolmetscher folgt in seinen Ausführungen konsequent dem FragestellungsModus und somit dem von dem Gesprächsleiter erzielten Sprecherwechsel und übernimmt (außer einmal) keine Gesprächsinitiative.

- Der Dolmetscher unterstützt durch seine Tätigkeit die klare Rollenverteilung im Gerichtssaal, auch durch zusätzliche Eigeninterventionen auf der syntaktischen Ebene, die er selbstverantwortlich einführt.

- Die Übertragung in die Zielsprache meistert er ohne jegliche Einwände des Gesprächsmoderators. Aus der Sicht des Gerichtes hat er seine Aufgabe erfolgreich durchgeführt.

Die diskursiv-linguistische Analyse zeigt allerdings, dass ein gewisses Potential zur Verbesserung der Dolmetschleistung vorhanden ist. Zum einen soll am eigenen Redestil gearbeitet werden, um unnötige Wiederholungen und zusätzliche Erklärungen zu vermeiden, es soll eine intensivere Reflexion betrieben werden, um eine mehr zielgerichtete und kompakte Verdolmetschung produzieren zu können und es soll ein Erfahrungsaustausch mit dem Gericht angestrebt werden, in dem auf die Adressierung sowie etwaige Probleme bei der Einspruchserhebung eingegangen werden könnte. Auf diese Weise könnten die in dieser Analyse festgestellten Mängel, in der Zukunft vermieden werden.

Die Ergebnisse dieser Studie dürfen nicht als repräsentativ angesehen werden, da die Daten mittels Beobachtung und Aufnahme von lediglich einer Zeugenvernehmung erfasst wurden. Das Ziel der Untersuchung war es, eine Gesprächssituation hervorzuheben und näher zu betrachten sowie beispielhaft daraus Empfehlungen für mögliche Verbesserungen abzuleiten und aufzuzeigen. Eine jedenfalls erforderliche Zielsetzung künftiger Recherchen bestünde darin, diese Empfehlungen an einem weiteren Korpus abzufragen. Auch in dieser Rechtssache wurde der Dolmetscher im Rahmen einer weiteren Verhandlung wiederholt hinzugezogen. Es wäre also interessant zu überprüfen, inwiefern, sich der Dolmetscher in einer Kommunikationssituation, deren Inhalt ihm zum Teil bereits bekannt ist, zurechtfindet.

\section{Literatur}

Berk-Seligson, Susan (2002) The Bilingual Courtroom - Court Interpreters in the Judicial Process. Chicago/London: The University of Chicago Press.

BrINKER, Klaus/Sven F. SAGER (2001) Linguistische Gesprächsanalyse - Eine Einführung. Berlin: Erich Schmidt Verlag.

Coulthard, Malcolm (1985) An introduction to discourse Analysis. London: Longmann. 
DRESSLER, Malcom/Ruth WodAK (1989) Fachsprache und Kommunikation - Experten im sprachlichen Umgang mit Laien. Wien: Österreichischer Bundesverlag Gesellschaft. HALlidaY, Michael A.K./Hasan RUQAIYA (1994) Language, Context and Text: aspects of language in social-semiotic perspective. Oxford: Oxford University Press.

Hatim, Basil/Ian Mason (1947): Discourse and the Translator. New York: Longman.

HeNNE, Helmut/Helmut ReHBock (2001) Einführung in die Gesprächsanalyse. Berlin/New York: Walter De Gruyter.

HofFMANN, Lothar (1985) Kommunikationsmittel Fachsprache. Eine Einführung. Tübingen: Narr Verlag.

HofFMAN, Ludger (2007) „Die Wirklichkeit des Gerichts.“ Der Deutschunterricht 4, 36-50. Mai 2012. http://home.edo.uni-dortmund.de/ ${ }^{\sim}$ hoffmann/PDF/DU_Gericht.pdf/.

HyMes, Dell/John GuMPERz (1986) Directions in sociolinguistics: the ethnography of communication. Oxford: Basil Blackwell.

KADRIĆ, Mira (2006) Dolmetschen bei Gericht. Wien: Facultas Verlag.

Kalina, Sylwia (1998) Strategische Prozesse beim Dolmetschen: theoretische Grundlagen, empirische Fallstudien, didaktische Konsequenzen. Tübingen: Narr Verlag.

LidDICOAT, Anthony J. (2007) An introduction to Conversation Analysis. London: Continuum.

MikKeLson, Holly (2000) Introduction to court interpreting. Manchester: St. Jerome Publishing.

MikKeLson, Holly (1998) „Awareness of the Language of the Law and the Preservation of Register in the Training of Legal Translators and Interpreters." In: I. G. Izquierdo/J. Verdegal (Hg), Los estudios de traducción: Un reto didáctico. Valencia: Universitat Jaume I, 87-100.

Möhn, Dieter/Roland Pelka (1984). Eine Einführung. Tübingen: Niemeyer.

OKSAAR, Els (1988) Fachsprachliche Dimensionen. Tübingen: Narr Verlag.

Pfeiffer, Oskar E./Ernst Strounal (1989) Das Gesetz aus dem Baukasten - Zur Herstellung von Wirklichkeit durch juristische Sprachschablonen. Wien: Österreichischer Bundesverlag Gesellschaft.

SeIBERT, Thomas-M. (2012) „Der Gerichtssaal.“ Rechtssemiotik. Mai 2012. http://www.rechtssemiotik.de/de/sachen/gerichtssaal_61259.shtml/

SchIFFrIN, Deborah (1994) Approaches to Discourse. Oxford/Massachusetts: Blackwell Publishers.

StaneK, Malgorzata (2011) Dolmetschen bei der Polizei: Zur Problematik des Einsatzes unqualifizierter Dolmetscher. Berlin: Frank \& Timme.

VERDONIK, Darinka (2007): Jezikovni elementi spontanosti v pogovoru. Maribor: Slavistično društvo.

Von HaHn, Walther (1983) Fachkommunikation Entwicklung. Linguistische Konzepte. Berlin/New York: Walter de Gruyter

WisniowsKa, Marta (2010): „Verhandlungsdolmetschen und Übertragung des Verhandlungsablaufs." In: J. Maliszewski (Hg), Posener Beiträge zur Germanistik Diskurs und Terminologie beim Fachübersetzen und Dolmetschen. Frankfurt am Main: Peter Lang, 151-167. 


\section{Transkription}

R 00:25:10 Najprej bi gospoda vprašal, eh, + + kaj če in kaj mu je znano glede pogodbe med firmo STUHAUS in, eh, najprej seveda Markom Horvatom, potem pa med firmo Efekt, eh, firmo Babilon, in pa, eh, Topič Tanjo in pa Nevenko Žigart.

D 00:26:00 + + Zuerst möchte ich Sie, Herr Müller, fragen, ob Ihnen was bekannt ist, beziehungsweise, was ist Ihnen bekannt, bezüglich der Verträge, zwischen der Firma oder dem Unternehmen, STUHAUS, auf der einen Seite und auf der anderen Seite, zuerst Marko Horvat und dann nachträglich der Gesellschaften EFEKT, BABILON und dann in der Folge noch die natürlichen Personen Tanja Topič und Nevenka Žigart. +

Z 00:51:00 Also ich möchte betonen, dass ich mit März 2011 in das Unternehmen eingetreten bin. Ich habe den Akt quasi übernommen, ich kenne die Mietverträge, + + sowohl von Efekt als jetzt im Anschluss auch mit Babilon. $\mathrm{Mmm}++$ meine Aufgabe hat insofern begonnen, als ich einen Akt auf den Tisch bekommen habe mit großen offenen Posten. Ich habe mir die Mietverträge angesehen, ich habe im Anschluss auch mit dem Herrn Žigart persönlich gesprochen., ich habe das Protokoll von meinem Vorgänger gelesen. Das wurde mir quasi präsentiert, das ist der Letztstand, basierend auf diesem müssen jetzt die weiteren Maßnahmen besprochen werden.

D 00:54:40 + + Rad bi poudaril, da sem marca leta 2011 pravzaprav se pridružil, eh, podjetju in da sem $v$ tem trenutku $v$ bistvu prevzel to zadevo oziroma, mi je bila predana od predhodnika, eh, tako, da sem seznanjen z najemnimi pogodbami, tako $\mathrm{s}$ tisto pogodbo, ki je bila sklenjena s podjetjem EFEKT kot tudi z potem naslednje, z BABILONOM. Moja naloga, ko sem prevzel to funkcijo, je bila ta, da sem v bistvu dobil ta odprti spis na mizo, moj-, preštudiral sem te najemne pogodbe, ki obstajajo. Moj predhodnik mi je v bistvu dejal, da pač je zdaj tukaj potrebno sprejeti nadaljnje ukrepe. Govoril sem tudi osebno z osebo Žigart in si v bistvu ogledal zapisnik, ki je bil sestavljen. Torej, kot rečeno, povedano mi je bilo, da je to zdaj zadnje stanje stvari, in da naj od tukaj ukrepam naprej. +

R 00:06:40 Če nam lahko pove prosim, eh ++, kdo je bil njegov predhodnik? Če ga lahko imenuje?

D 00:03:50 Wer war Ihr Vorgänger? Könnten Sie den Namen nennen?

Z 00:13:10 Das, eh, mein direkter Vorgänger war die Frau Magister Ekarim. Es ist aber so innerhalb des Konzernes, dass wir immer die Zustimmung der Geschäftsführung brauchen, aus dem jeweiligen Fachbereich.

D 00:19:70 No, moja nepos v.. + moja predhodnica je bila gospa magister Paula Ekarim, vendar je pri nas znotraj koncerna tako, da velja pravilo, da za vse postopke, ki jih vodimo, potrebujemo potrdilo predstojnika, torej oddelka, ali poslovodje našega, torej nadrejenega.

R 00:06:50 Že, ampak zanima me, kdo se je pred gospodom ukvarjal s temi pogodbami, s tem konkretnim spisom?

D 00:04:50 Wer hat sich also konkret vor Ihnen mit dieser Akte und mit diesen Mietverträgen beschäftigt?

Z 00:05:70 Die Frau Magister Ekarim und reportet hat sie an den Herrn Magister Bernhardt.

D 00:05:40 Gospa magister Ekarim, poročala pa je gospodu Bernhardt. 
R 00:15:00 Aha, zdaj, glede na to, da je gospod prevzel ta spis šele v mesecu marcu letošnjega leta, me zanima, eh, kaj se je pogovarjal z gospo Žigartovo?

D 00:12:00 + + Hinsichtlich der Tatsache, dass Sie in März dieses Jahres die Akte beziehungsweise diesen Fall übernommen haben, würde mich interessieren, worüber Sie mit Frau Žigart gesprochen haben?

Z 00:07:60 Also ich habe mit Herrn Žigart gesprochen, Frau Žigart, wäre mir nicht bekannt. Das sagte ich schon.

D 00:04:80 Govoril sem z gospodom Žigartom. Gospe Žigart ne poznam.

R 00:04:20 Mhm. Lahko pove mogoče ime gospoda Žigarta?

D 00:03:10 Könnten Sie den Namen von Herrn Žigart nennen? + Den Namen

Z 00:02:20 Žigart.

D 00:02:50 + Žigart ist der Nachname, den Vornamen.

Z 00:01:20 Nein.

D 00:01:60 Ne, ne vem.

R 00:08:70 Mhm. Eh, nam lahko poveste mogoče vsebino razgovora. Je šlo o tem predmetu, ali o čem drugem?

D 00:08:50 Könnten Sie uns den Inhalt dieses Gespräches darlegen? Ging es um diese Sache, oder haben Sie über etwas anderes gesprochen?

Z 00:37:30 Es ging ausschließlich um diese Sache. Meine Aufgabe und meine Arbeit besteht auch darinnen, ++ darin, dass wir offene Posten einbringlich machen. Wir haben Verträge, an diese ist sich zu halten und meine Aufgabe war es, eh, weil im letzten Protokoll vermerkt war, dass noch ein Folgetermin in Maribor stattfinden sollte, mein Vorgesetzter hat empfohlen diesen Folgetermin wahrzunehmen. + +Und betreffend dieses Folgetermins habe ich mit Herrn Žigart Kontakt aufgenommen.

D 00:44:90 Ja, eh, + + torej seveda sem govoril z njim o tej zadevi. Moje delo med drugim, ali moje naloge, obseg nalog med drugim vključuje tudi to, da preverim, ali so kje kakšne odprte postavke in da te odprte postavke seveda na nek način poskušam, + + izterjati lahko bi rekli, ehm, in da poskrbim za to, oziroma preverim, ali se naše stranke držijo dogovorov, ki so bili pač v pogodbah usklajeni. No, v moji zadevi je bilo predvideno, da bo se zgodil še en + + termin, za večimi, ki so zgodili že pred tem v Mariboru, in moj šef mi je pravzaprav svetoval, naj grem na ta sestanek oziroma, naj se tega termina udeležim, zato sem tudi kontaktiral z gospodom Žigartom.

R 00:06:00 + + Mi še vedno ne vemo, o čem je kontaktiral, o čem se je pogovarjal?

D 00:04:00 Gut. Wir wissen noch immer nicht, worüber haben Sie mit ihm gesprochen?

Z 00:14:00 Ich habe ihm gesagt, er soll umgehend die Miete bezahlen. Dass, eh, er sich an seine vertraglichen Verpflichtungen zu halten hat, ansonsten wir uns gezwungen sähen rechtliche Maßnahmen in die Wege zu leiten.

D 00:14:40 + + vRekel sem mu, naj plača najemnino, rekel sem mu, da je dolžan spoštovati dogovor iz naše pogodbe, sicer bom zoper njega uvedel sodni postopek.

R 00:07:80 Mhm. Zanima me, ali, + + oziroma, kako je gospod Žigart na to reagiral?

D 00:02:90 Wie hat Herr Žigart darauf reagiert? 


\begin{tabular}{|c|c|c|}
\hline $\mathrm{Z}$ & $00: 31: 10$ & $\begin{array}{l}\text { Er hat angefangen, unter anderem über die Investitionen zu sprechen, + + ich } \\
\text { habe gesagt die Investitionen, das stand auch schon im Protokoll zur Sache, es } \\
\text { steht auch im Mietvertrag drinnen, dass Investitionen nicht abgegolten werden. } \\
\text { Für mich als Vermieter, ich kann mit einer Discobar nichts anfangen. Die stellt } \\
\text { für mich keinerlei Wert dar. Ich habe gesagt, wenn er jetzt nicht bezahlt, dann } \\
\text { werde ich die Sache dem Anwalt übergeben. }\end{array}$ \\
\hline $\mathrm{D}$ & $00: 40: 30$ & $\begin{array}{l}\text { + + Gospod Žigart je med drugim začel omenjati določene investicije, ki jih naj } \\
\text { bi izvajal, eh, povedal sem mu, da je to bilo zapisano že tudi v zapisniku enkrat } \\
\text { pred tem in da tudi iz naše medsebojne najemne pogodbe izhaja, da se } \\
\text { kakršnekoli investicije, eh, torej niti o tem ni bilo dogovora, niti se ne plačujejo. } \\
\text { Zame kot najemodajalca nek disco bar ali neka diskoteka praktično nima vred- } \\
\text { nosti, zato tudi te investicije seveda niso v interesu. In povedal sem mu, da če } \\
\text { ne bo plačal, bom vključil odvetnika. }\end{array}$ \\
\hline $\mathrm{AB}$ & & Smem iti za trenutek ven? \\
\hline $\mathrm{R}$ & & Prav. Ja, ja, seveda lahko greste. \\
\hline $\mathrm{Z}$ & 00:01:60 & Wenn ich kurz noch was sagen darf? \\
\hline $\mathrm{D}$ & 00:01:20 & Če smem še nekaj dodati? \\
\hline $\mathrm{Z}$ & $00: 25: 90$ & $\begin{array}{l}\text { Es ist, }++ \text { ich war am, }++ \text { Anfang April auch in Maribor. Ich habe einen } \\
\text { gemeinsamen Gesprächstermin vorgeschlagen und denn hat der Herr Žigart } \\
\text { abgelehnt. Also er ist einfach kurzfristig abgesagt und daraufhin, ich hab das } \\
\text { persönliche Gespräch angeboten, bin zurückgewiesen worden, }++ \text { wir bekom- } \\
\text { men keine Zahlungen, damit war die Sache für mich klar, dass diesen Fall } \\
\text { weiter Herr Škrjanec betreuen muss. }\end{array}$ \\
\hline $\mathrm{D}$ & $00: 25: 80$ & $\begin{array}{l}\text { + + No povedal bi še, da sem bil v začetku meseca aprila v Mariboru. Predlagal } \\
\text { sem še en sestanek. No gospod Žigart me je v bistvu zavrnil oziroma je kratko- } \\
\text { ročno ta sestanek odpovedal, tako da z mojega stališča v bistvu lahko rečem, } \\
\text { predlagal sem osebni razgovor, v katerem bi se stvari še lahko pogovorile, plačil } \\
\text { ni bilo in zame je bilo v tem trenutku jasno, da bo moral zadevo od tu naprej } \\
\text { prevzeti odvetnik Škrjanec. }\end{array}$ \\
\hline $\mathrm{R}$ & 00:02:80 & d odvetnik, izvolite. Vprašanja. \\
\hline A1 & $00: 26: 30$ & $\begin{array}{l}\text { Gospod Müller, ahm, ali ste vi osebno komurkoli od štirih toženih strank kda- } \\
\text { jkoli rekli, da lahko investira, oziroma ali ste jim kakorkoli dali vedet, da bodo } \\
\text { investicije poplačane, }++ \text { vrnjene z vaše strani? }\end{array}$ \\
\hline $\mathrm{D}$ & $00: 26: 60$ & $\begin{array}{l}\mathrm{Z}^{+}+\text {Herr Müller, haben sie persönlich, irgendeinmal, einer von den vier } \\
\text { beklagten Parteien gesagt, dass sie Investitionen tätigen können } \\
\text { beziehungsweise dürfen oder beziehungsweise haben sie ihnen gegenüber } \\
\text { gesagt, dass getätigte Investitionen von Ihrer Seite }++ \text { also erstattet werden, } \\
\text { zurückgezahlt werden? }\end{array}$ \\
\hline $\mathrm{Z}$ & 00:02:30 & Nein, das kann ich definitiv verneinen. \\
\hline $\mathrm{D}$ & 00:02:70 & $\mathrm{Ne}$, na to lahko definitivno odgovorim z ne. \\
\hline A1 & $00: 21: 30$ & $\begin{array}{l}\text { Vprašal bi vas potem, ali vam je + + znano, da bi to naredil vaš predpostavljeni, } \\
\text { gospod Bernhardt, namreč, da bi se dogovoril s katerokoli od toženih strank, }+ \\
+ \text { da bodo investicije povrnjene? }\end{array}$ \\
\hline
\end{tabular}


D 00:17:10 + Des Weiteren möchte ich Sie fragen, ob Ihnen bekannt ist, dass Ihr Vorgesetzter, Herr Bernhardt eine derartige Absprache über die Zurückzahlung, eh Erstattung der getätigten Investitionen mit einer der vier beklagten Parteien getroffen hätte?

Z 00:15:50 Mir ist eine solche Vereinbarung nicht bekannt und Herr Bernhardt hat auch mir gegenüber auch betont, dass er eine solche Zusage nie getätigt hat. Hätte auch keinerlei Sinn für uns als Firma.

D 00:18:80 + + Ni + mi znano, da bi gospod Bernhardt tak dogovor sklenil, tudi gospod Bernhardt je v relaciji do mene povedal, da takšnega soglasja zagotovo ni dal, to tudi z naše strani, s strani najemodajalca ne bi bilo smiselno.

A1 00:07:80 Isto vprašanje velja za gospo Paulo Ekarim.

D 00:08:30 Es gilt jetzt nochmal die gleiche Frage, bezogen nicht auf Ihren Vorgesetzten, sondern auf Paula Ekarim.

Z 00:19:20 Mir ist nicht bekannt, dass Frau Ekarim eine Zusage gemacht hätte. Und selbst für den Fall, den ich hier dezidiert ausschließen möchte, dass sie eine Zusage gemacht hätte, wäre sie nicht berechtigt gewesen diese zu machen, ohne Zustimmung der Geschäftsführung.

D 00:24:20 Ni mi znano, da bi gospa Paula Ekarim dala takšno soglasje in tudi v primeru, ki pa ga želim na tem mestu še enkrat izrecno zanikati, ampak tudi v primeru če bi to bilo narejeno, če bi to soglasje z njene strani bilo dano, za to ne bi bila upravičena, kajti za to bi potrebovala soglasje nadrejenega.

\begin{tabular}{|lrl|}
\hline A1 & $00: 01: 60$ & Lepa hvala \\
D & $00: 01: 40$ & Danke schön. \\
\hline R & Če mate vi kakšno vprašanje? (ZU DER BEKLAGTEN, DA DER ANWALT \\
& DEN VERHANDLUNGSSAAL VERLASSEN HAT) \\
\hline B & Prosim? \\
\hline R & Če imate kakšno vprašanje? \\
\hline B & Ja, tu mi je napisal. \\
\hline B & Ali ste prijavili predmetno potrditev v stečajni postopek proti Efektu? \\
\hline D & Haben Sie ...? \\
\hline R & Samo malo (UNTERBRICHT DIE VERDOLMETSCHUNG MIT EINER \\
\hline B & HANDBEWEGUNG). Terjatev je mislil. Izvolite. \\
\hline A1 & Ja. \\
\hline R & Ugovarjam vprašanju, ni v relevantni zvezi s to zadevo. \\
\hline D & Sprejmemo sklep, vprašanje se ne dovoli. Naprej. \\
\hline B & Es wird der Beschluss gefasst, die Frage wird nicht gestattet. \\
\hline D & Ali je bila priznana? \\
\hline AB & Wurde diese anerkannt? \\
R & Isto. \\
B & O0:06:20 & Ali ste kontaktirali z gospodom ali z gospo Žigart, + + osebno? \\
& \\
\hline
\end{tabular}


D 00:05:00 Haben Sie mit Herrn oder mit Frau Žigart persönlich Kontakt aufgenommen?

\begin{tabular}{|c|c|}
\hline $\mathrm{AB}$ & Priča je že odgovorila na to vprašanje. \\
\hline $\mathrm{R}$ & Sprejmemo sklep, vprašanje se ne dovoli. \\
\hline $\mathrm{D}$ & Der Zeuge hat die Frage bereits beantwortet \\
\hline $\mathrm{R}$ & Naprej. \\
\hline $\mathrm{D}$ & Es wird der Beschluss gefasst, die Frage wird nicht gestattet. \\
\hline B & Glede na to, da ste s podpisom dne $6.4 .2011,++$ \\
\hline $\mathrm{D}$ & Hinsichtlich der Tatsache, dass Sie am 6.4.2011 mit Ihrer Unterschrift ... \\
\hline B & $\begin{array}{l}\text { obračun, }++ \text { čakaj, kaj to piše, obračun naložba Žigarta? Ne, kak to zdaj? Da } \\
\text { ste z podpisom + + dne, }++ \text { obračun na gospoda Žigarta + + kaj zaj to pomeni? }\end{array}$ \\
\hline $\mathrm{D}$ & Die Frage ist inkonsistent. \\
\hline $\mathrm{R}$ & Zdaj, + če ne znate, + bom rekel, nekako, vprašanja postavit, potem..? \\
\hline B & $\begin{array}{l}\text { Čakajte, malo, ja+ +? Zaj + + to je napisal + + glede na to, da ste z gospodom z } \\
\text { dne 6.4.2011 obračun, + + na gospoda Žigarta. Tak piše. }\end{array}$ \\
\hline A1 & Moram ugovarjati, sodnik? \\
\hline $\mathrm{R}$ & Ne, vprašanje ni razumljivo, tak da + + mate kakšno drugo vprašanje? \\
\hline $\mathrm{D}$ & Die Frage war unverständlich. \\
\hline B & Ali ste izdali iz knjigovodske listine glede terjatev in glede porokov? \\
\hline $\mathrm{D}$ & Ob Sie Buchhaltungsurkunden ++ \\
\hline $\mathrm{R}$ & Kakšnih porokov? \\
\hline B & Ne vem, tak piše? \\
\hline $\mathrm{R}$ & Če je izdal $++?$ \\
\hline B & Knjigovodske listine. ++ Glede terjatev. ++ Globe porokov. \\
\hline A1 & Vprašanje nima trditvene podlage in predlagamo, da se zavrne. \\
\hline $\mathrm{D}$ & $\begin{array}{l}\text { Die Frage hat keinen Behauptungsgrund. Es wird beantragt, dass sie zurück- } \\
\text { gewiesen wird. }\end{array}$ \\
\hline $\mathrm{R}$ & Sprejmemo sklep: Vprašanje se ne dovoli. Imate kakšno drugo vprašanje? \\
\hline $\mathrm{D}$ & Es wird der Beschluss gefasst, die Frage wird nicht gestattet. \\
\hline B & Ne. \\
\hline $\mathrm{R}$ & Prav, za pričo ni več vprašanj. + + Snemanje prekinemo. \\
\hline
\end{tabular}

\section{LEGENDE ZUR TRANSKRIPTION}

$\begin{aligned}+ & \text { Pause } \\ ++ & \text { längere Pause } \\ \mathrm{R} & \text { Richter } \\ \mathrm{D} & \text { Dolmetscher } \\ \mathrm{Z} & \text { Zeuge } \\ \mathrm{B} & \text { Beklagte }\end{aligned}$




\author{
AK Anwalt des Klägers \\ $\mathrm{AB} \quad$ Anwalt der Beklagten \\ Wortlaut (grau unterlegt) gleichzeitiges Sprechen mehrerer Gesprächsteilnehmer \\ $\&$ simultanes Flüsterdolmetschen \\ Wortlaut (grau unterlegt \& simultanes Flüsterdolmetschen \\ Kursivschrift) \\ Wortlaut (Kursivschrift) schlechter hörbare Wörter oder Passagen

\section{Zusammenfassung \\ EMPIRISCHE ANALYSE EINER VERDOLMETSCHTEN GERICHTSVERHANDLUNG AUS DEM SLOWENISCHEN INS DEUTSCHE}

Dieser Artikel befasst sich mit dem Diskurs in einer Gerichtsverhandlung mit verschiedensprachigen Parteien. Die übliche Konversation im Gerichtssaal findet in Form eines Dialogs statt, wobei die Strategie des Gespräches durch den Richter als Gesprächsmoderator determiniert wird. Die Interaktion im gedolmetschten Diskurs verläuft anders als im nicht gedolmetschten Diskurs. Bei gedolmetschten Verhandlungen übernimmt der Gerichtsdolmetscher, als ein Mittler in der Konversation, die verantwortungsvolle Aufgabe, die richterliche Strategie der Konversation, also den eigentlichen Verhandlungsablauf, sinngemäß und originaltreu in den zielsprachlichen Kontext zu transportieren. Meistert er seine Aufgabe, wird ein reibungsloser Kommunikationsablauf im Sinne der gegenseitigen Einflussnahme der jeweiligen Kommunikationsteilnehmer gewährleistet. Scheitert er bei seinem Vorhaben, so kommt es in der Kommunikation zu Störungen, die durch zusätzliche sprachliche Interventionen beseitigt werden müssen. Seine Tätigkeit wird determiniert und mitbeeinflusst von den Unterschieden in der Rechtsterminologie der beiden Sprachen (Sprachregister), von dem vorgegebenen Dolmetschmodus, und von der Gesprächsstrategie des Sprachmoderators. Somit ist das Ziel des vorliegenden Beitrags, anhand einer empirischen Analyse einer gedolmetschten Zeugenvernehmung herauszufinden, inwiefern es dem Gerichtsdolmetscher gelingt, den ausgangssprachlichen Diskurs originaltreu in die Zielsprache zu übertragen und auf welche Probleme er bei diesem Übertragungsprozess stößt. Der vorliegenden Untersuchung dienen die von den Gerichten veranlassten Audioaufnahmen von mündlichen Verhandlungen als Korpus.

Schlüsselwörter: Diskurs, Fachsprache, Rechtssprache, Vernehmung, Gerichtsdolmetschen, Gesprächsanalyse.

\title{
Povzetek \\ EMPIRIČNA ANALIZA SODNEGA TOLMAČENJA IZ SLOVENŠČINE V NEMŠČINO
}

Prispevek obravnava diskurz v sodni obravnavi, v katerem sodelujejo stranke, ki govorijo različne jezike. Pogovor v sodni dvorani običajno poteka kot dialog, strategijo govora pa določa sodnik, ki prevzema vlogo moderatorja. Interakcija v diskurzu s tolmačenjem se razlikuje od tiste, pri kateri tolmačenje ni potrebno. $\mathrm{V}$ tolmačenih obravnavah prevzema tolmač, kot po- 
srednik v konverzaciji, odgovorno nalogo, da sodnikovo strategijo vodenja pogovora, torej dejanski verbalni potek obravnave, smiselno in skladno z izvirnikom prenese v ciljni jezik. Če je pri tem uspešen, je zagotovljen nemoten potek komunikacije in možnost, da udeleženci pogovora medsebojno vplivajo drug na drugega. V nasprotnem primeru pride v komunikaciji do motenj, ki jih je treba odpraviti s pomočjo dodatnih jezikovnih intervencij. Na dejavnost tolmača dodatno vplivajo izbrani modus tolmačenja, razlike v pravnih terminologijah izvirnega in ciljnega jezika (jezikovni register) in moderatorjeva strategija vodenja pogovora v sodni dvorani. Cilj tega prispevka je s pomočjo empirične analize zaslišanja priče s pomočjo tolmača ugotoviti, do kakšne mere sodni tolmač pri prenosu diskurza iz izhodiščnega v ciljni jezik ostaja zvest izvirniku in na kakšne probleme pri tem naleti. Kot korpus za raziskavo služijo avdio posnetki ustnih obravnav, ki jih hrani sodišče.

Ključne besede: diskurz, strokovni jezik, pravni jezik, zaslišanje, sodno tolmačenje, analiza govora. 